\title{
Observer-based sampled-data controller of linear system for the wave energy converter
}

\author{
Geun Bum Koo ${ }^{1}$, Jin Bae Park $^{1}$ and Young Hoon Joo ${ }^{2}$ \\ 1 Department of Electrical and Electronic Engineering, Yonsei University, Seoul, 120-749, Korea \\ 2 Department of Control and Robotics Engineering, Kunsan National University, Kunsan, Chonbuk, 573-701, \\ Korea
}

\begin{abstract}
In this paper, an observer-based sampled-data controller of linear system is proposed for the wave energy converter. Based on the sampled-data observer, the controller is design. In the closed-loop system with controller, it obtains the norm inequality between the continuous-time state variable and the discrete-time one. Using the norm inequality, sufficient condition is derived for the asymptotic stability of the closed-loop system and formulated in terms of linear matrix inequality. Finally, the wave energy converter simulation is provided to verify the effectiveness of the proposed technique.
\end{abstract}

Key Words: Observer-based, sampled-data controller, linear matrix inequality, wave energy converter

\section{Introduction}

Recently, according as the renewable energy has attracted much attention, the wave energy which is one of the renewable energies and its converter are continuously studied. The wave energy converter which is generated by the vertical motion of the wave has bright prospect, because the wave energy is a stable and rich energy source. However, as the wave energy converter is generated in the sea, the analog control or estimation of the wave energy converter is not possible. So, the remote digital control or estimation technique is necessary in the wave energy converter.

The sampled-data control technique which is one of the digital control techniques has obtained remarkable results[1, 2, 3, 4, 5, 6, 7, 8] and can be classified into few categories. The typical technique is to design the digital controller of the discrete-time system using the discretization of the original analog system. But, this technique is too hard to apply into multifarious systems such as the nonlinear system and the time-delay system.

To solve the above problem, many studies have been progressed. In [2, 3, 4], the sampled-data fuzzy controller is converted into a time delay controller in the continuoustime sense. Chang[5] and Lee[6, 7] presented the global intelligent digital redesign (IDR) methods based on the ge-

Manuscript received Oct. 30, 2011; revised Nov. 22, 2011; accepted Nov. 29, 2011.

This work was supported by the New and Renewable Energy of the Korea Institute of Energy Technology Evaluation and Planning(KETEP) grant funded by the Korea government Ministry of Knowledge Economy. [20103020070070] netic algorithms and the convex optimization approaches. However, there were no studies of the observer-based sampled-data controller for the wave energy converter.

In this paper, we propose the observer-based sampleddata control technique for the linear system. For the sampled-data controller design, we design the sampleddata observer and observer-based controller is proposed. Based on the closed-loop system with observer and the controller, it obtains the norm inequality between original continuous-time state variable and its discrete-time one. Sufficient conditions are investigated for the asymptotic stability of the state variable and the estimation error. In the sense of Lyapunov, their constructive design condition is presented in terms of linear matrix inequality. By simulation for the wave energy converter, it shows the validity of the proposed techniques.

This paper is organized as follows: Section 2 describes the linear system and the observer-based controller of interest. The stability condition for the closed-loop system with sampled-data output is proposed in Section 3. In Section 4, a numerical example is given for illustration. Finally, the conclusions are given in Section 5.

\section{Preliminaries}

Consider a linear system with sampled-data output as follows:

$$
\begin{aligned}
& \dot{x}(t)=A x(t)+B u(t) \\
& y(t)=y(k T)=C x(k T)
\end{aligned}
$$


where $x(t)$ is the state variable; $u(t)$ is the input variable; and $y(k T)$ is the sampled-data output variable for $t \in[k T, k T+T), k \in \mathbb{Z}_{\geq 0} ; T \in \mathbb{R}_{>0}$ is a given sampling period; $A, B$ and $C$ denote nominal system matrices with appropriate dimensions.

Based on the system (1), we consider the observer-based sampled-data controller in the following form:

$$
\begin{aligned}
& \dot{\hat{x}}(t)=A \hat{x}(t)+B u(t)+L(y(t)-\hat{y}(t)) \\
& \hat{y}(t)=\hat{y}(k T)=C \hat{x}(k T) \\
& u(t)=u(k T)=K \hat{x}(t)
\end{aligned}
$$

where $\hat{x}(t)$ is an estimation of state variable, $L$ and $K$ are the observer and controller gains, respectively.

Consider the estimation error as follows:

$$
e(t)=x(t)-\hat{x}(t)
$$

Substituting (2) into (1) and (3), we obtain the following closed-loop system:

$$
\begin{aligned}
{\left[\begin{array}{c}
\dot{x}(t) \\
\dot{e}(t)
\end{array}\right]=} & {\left[\begin{array}{cc}
A+B K & -B K \\
0 & A-L C
\end{array}\right]\left[\begin{array}{l}
x(k T) \\
e(k T)
\end{array}\right] } \\
& +\left[\begin{array}{cc}
A & 0 \\
0 & A
\end{array}\right]\left[\begin{array}{c}
x(t)-x(k T) \\
e(t)-e(k T)
\end{array}\right] .
\end{aligned}
$$

The objective of this paper is to obtain the stability condition of the closed-loop system (4).

\section{Main results}

To guarantee the stability of the closed-loop system (4), we need the following lemmas and proposition.

Lemma 3.1 ([9]). Given any function vector $x$, matrix $P=$ $P^{T} \succ 0$, and $t_{0}, t_{f} \in \mathbb{R}$ with $t_{0}<t_{f}$, we have

$$
\begin{aligned}
& \left(\int_{t_{0}}^{t_{f}} x(\tau) d \tau\right)^{T} P\left(\int_{t_{0}}^{t_{f}} x(\tau) d \tau\right) \\
& \leq\left(t_{f}-t_{0}\right) \int_{t_{0}}^{t_{f}} x(\tau)^{T} P x(\tau) d \tau
\end{aligned}
$$

Lemma 3.2. [10] Given constant symmetric matrices $N$, $O$ and $L$ of appropriate dimensions, the following inequalities

$$
O>0, \quad N+L^{T} O L<0
$$

are equivalent to the following inequality

$$
\left[\begin{array}{cc}
N & L^{T} \\
L & -O^{-1}
\end{array}\right]<0 \quad \text { or } \quad\left[\begin{array}{cc}
-O^{-1} & L \\
L^{T} & N
\end{array}\right]<0 .
$$

Proposition 3.3. In (4), there exists some constant $\delta>0$ such that

$$
\left\|\left[\begin{array}{l}
x(t) \\
e(t)
\end{array}\right]\right\| \leq \delta\left\|\left[\begin{array}{l}
x(k T) \\
e(k T)
\end{array}\right]\right\|
$$

where

$$
\begin{aligned}
\delta & =(1+T\|\Phi\|) e^{\|A\| T}, \\
\Phi & =\left[\begin{array}{cc}
B K & -B K \\
0 & -L C
\end{array}\right] .
\end{aligned}
$$

The main result is summarized in the following theorem.

Theorem 3.4. If there exist some symmetric and positive definite matrices $P$ and $Q$, some matrices $M$ and $N$, such that the following LMIs are satisfied, then the state variable and estimation error (4) are asymptotically stable.

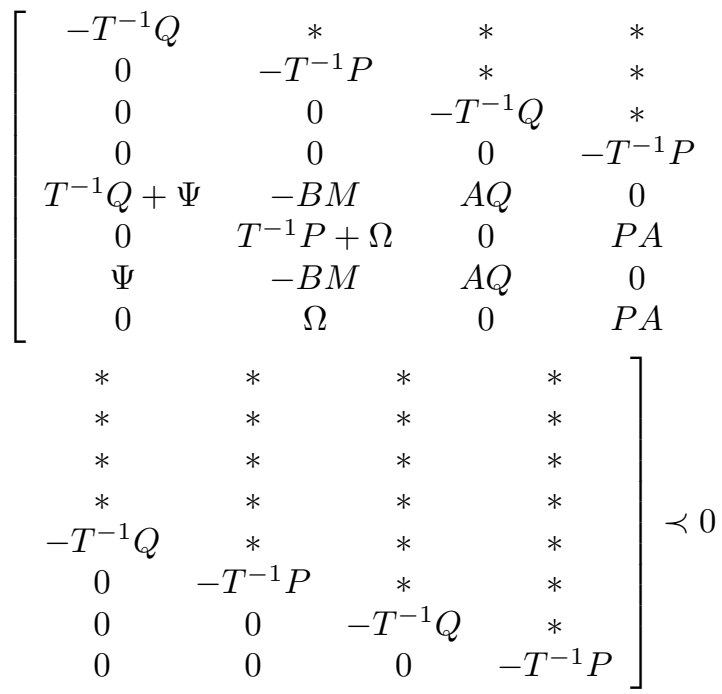

where

$$
\begin{aligned}
& \Psi=A Q+B M, \\
& \Omega=P A-N C
\end{aligned}
$$

and $*$ denotes the transposed elements in the symmetric positions.

Proof. Consider the exact discretized model of the closedloop system (4) in the following form:

$$
\begin{aligned}
\chi(k T+T)= & \chi(k T) \\
& +\int_{k T}^{k T+T} \Theta \chi(k T)+\Lambda(\chi(t)-\chi(k T)) d t
\end{aligned}
$$

where

$$
\begin{aligned}
\chi(k T) & =\left[\begin{array}{l}
x(k T) \\
e(k T)
\end{array}\right], \\
\Theta & =\left[\begin{array}{cc}
A+B K & -B K \\
0 & A-L C
\end{array}\right], \\
\Lambda & =\left[\begin{array}{ll}
A & 0 \\
0 & A
\end{array}\right] .
\end{aligned}
$$


Based on the discretized model (7), we consider Lyapunov functional candidate as follows:

$$
V(\chi(k T))=\chi(k T)^{T} P \chi(k T) .
$$

Then, if the difference of (8) is the negative value, the closed-loop system (4) satisfies an asymptotic stability by Proposition 1. The difference of (8) becomes

$$
\begin{aligned}
& \Delta V(\chi(k T)) \\
& =\chi(k T+T)^{T} P \chi(k T+T)-\chi(k T)^{T} P \chi(k T)
\end{aligned}
$$

Substituting (7) into (9) and using Lemma 1, we have

$$
\begin{aligned}
\Delta & V(\chi(k T)) \\
\leq & T \int_{k T}^{k T+T}\left(\left(\frac{1}{T} I+\Theta\right) \chi(k T)+\Lambda(\chi(t)-\chi(k T))\right)^{T} \\
& \times P\left(\left(\frac{1}{T} I+\Theta\right) \chi(k T)+\Lambda(\chi(t)-\chi(k T))\right) d t \\
& -\frac{1}{T} \int_{k T}^{k T+T} \chi(k T)^{T} P \chi(k T) d t \\
& -\frac{1}{T} \int_{k T}^{k T+T}(\chi(t)-\chi(k T))^{T} Q(\chi(t)-\chi(k T)) d t \\
& +T \int_{k T}^{k T+T}(\Theta \chi(k T)+\Lambda(\chi(t)-\chi(k T)))^{T} \\
& \times Q(\Theta \chi(k T)+\Lambda(\chi(t)-\chi(k T))) d t
\end{aligned}
$$

By using Lemma 2 and denoting $M=K Q$ and $N=P L$, the equation (10) is equivalent to the matrix of LMI (6). Thus, if the LMI (6) is satisfied, we can obtain the control gain $K$ and observer gain $L$ to stabilize the linear system with sampled-data output. The proof is complete.

Remark 3.5. This paper deals with the observer-based sampled-data controller for the linear system using the discretization. Because an analog system can be perfectly converted to the digital system in the linear system, the digital controller design for the discretized model is more simple than proposed method. But, in the nonlinear system case such as the fuzzy system, the proposed method is more valid.

\section{Simulation}

Consider an ocean wave energy harvesting application[12] with a linear permanent magnetic generator in the following form:

$$
\left[\begin{array}{l}
\dot{x}_{1}(t) \\
\dot{x}_{2}(t)
\end{array}\right]=\left[\begin{array}{cc}
0 & 1 \\
-\frac{\rho g A}{M+M_{a}} & 0
\end{array}\right]\left[\begin{array}{l}
x_{1}(t) \\
x_{2}(t)
\end{array}\right]+\left[\begin{array}{c}
0 \\
\frac{B^{2} N^{2} L^{2}}{M+M_{a}}
\end{array}\right] u(t)
$$

where $x_{1}(t)$ is the buoy displacement; $x_{2}(t)$ is the buoy velocity; $M$ is the mass of the buoy; $M_{a}$ is the added mass, $\rho$ is the density of sea water; $g$ is the gravitational acceleration; $A$ is the area of the buoy; $B$ is the magnetic flux density; $N$ is turns of the coil; $L$ is the coil length.

As shown in this system, there is no external disturbance. It means that the sea wave does not affect the wave energy converter. That is, this simulation shows the stability of the wave energy converter without sea wave.

The parameter values of the ocean wave energy harvesting application are determined in Table 1[13].

Table 1: The parameter values of the ocean wave energy harvesting application.

\begin{tabular}{|c|c||c|c|}
\hline Parameter & Value & Parameter & Value \\
\hline$M$ & $1344 \mathrm{~kg}$ & $M_{a}$ & $119.5 \mathrm{~kg}$ \\
\hline$\rho$ & $1030 \mathrm{~kg} / \mathrm{m}^{3}$ & $g$ & $9.8 \mathrm{~m} / \mathrm{s}^{2}$ \\
\hline$A$ & $4.86 \mathrm{~m}^{2}$ & $B$ & $1.4 T$ \\
\hline$N$ & 30 & $L$ & $2.64 \mathrm{~m}$ \\
\hline
\end{tabular}

Using LMI (6), the observer and controller gains are obtained:

$$
\begin{aligned}
K & =\left[\begin{array}{ll}
2.4500 & -0.4764
\end{array}\right], \\
L & =\left[\begin{array}{l}
28.2599 \\
48.1007
\end{array}\right] .
\end{aligned}
$$

The time responses of the ocean wave energy harvesting application and its observer are shown in Fig. 1 and 2, and the time responses of the estimation error are shown in Fig. 4 and 5 with $T=0.02$. As shown in these figures, we can know the performance of the observer-based sampled-data controller.

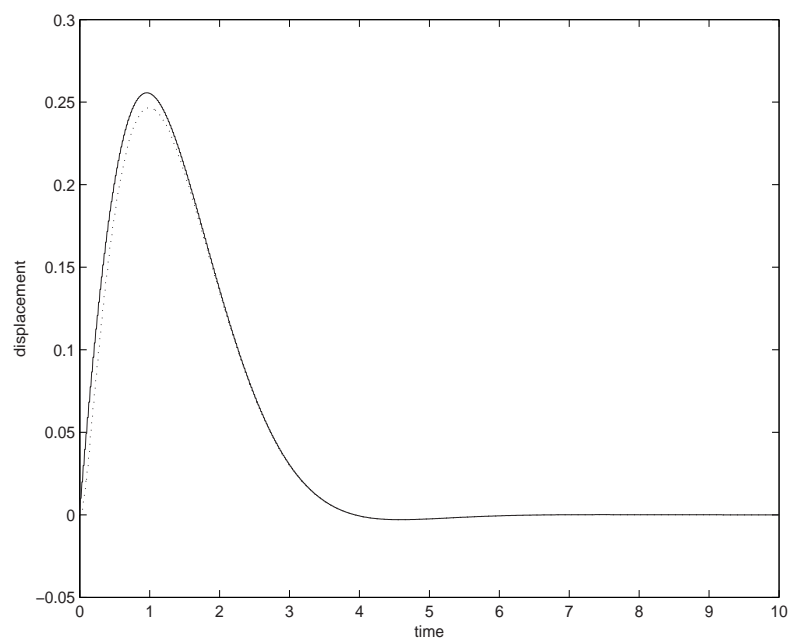

Figure 1: The displacement of the buoy and the observer: original (solid), observer (dotted) 


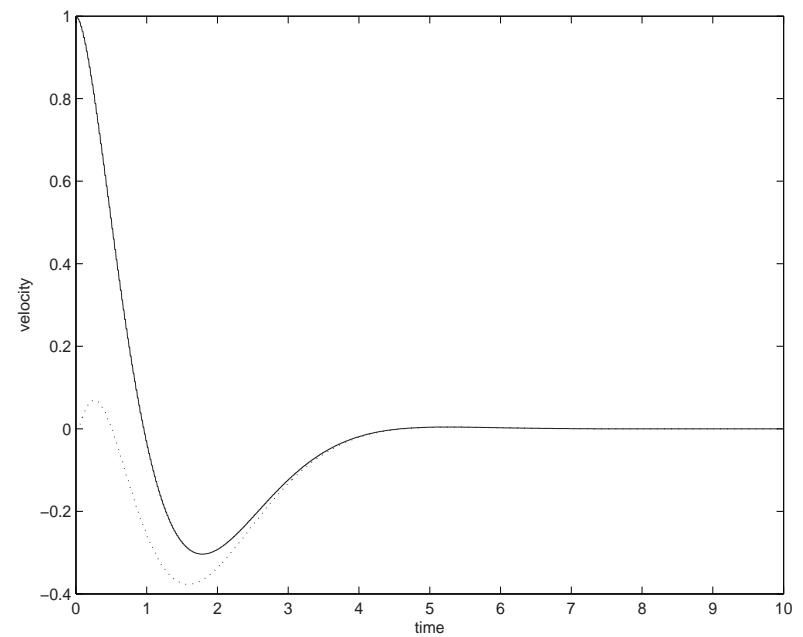

Figure 2: The velocity of the buoy and the observer: original (solid), observer (dotted)

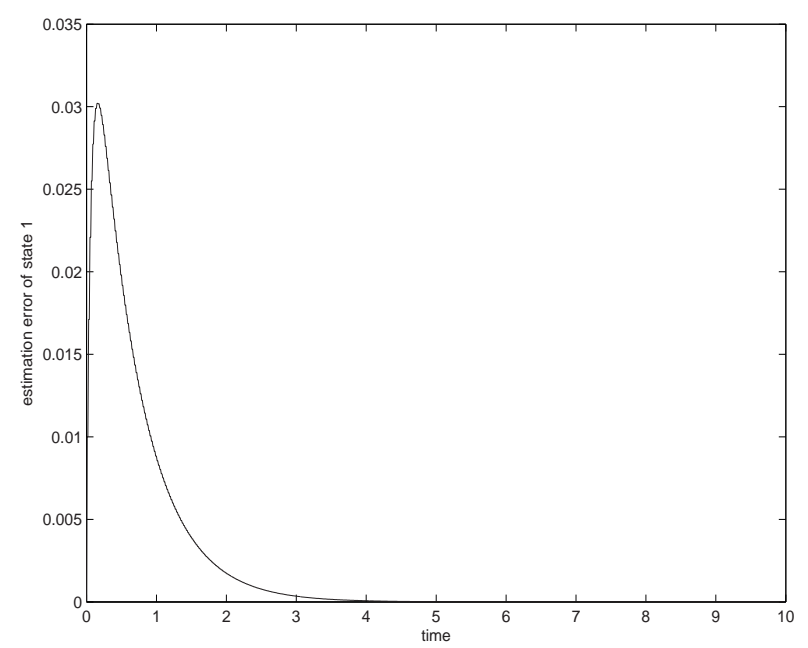

Figure 3: The estimation error of the displacement

\section{Conclusion}

In this paper, the observer-based sampled-data controller has been designed for the wave energy converter. The sampled-data controller is designed using the sampled-data observer and sufficient condition for asymptotic stability of the closed-loop system is designed in the linear matrix inequality format. Using the wave energy converter system, it has been shown to prove the advantage of the developed technique.

\section{References}

[1] Y. H. Joo, G. Chen and L. S. Shieh, "Hybrid state-space fuzzy model-based controller with dual-rate sampling

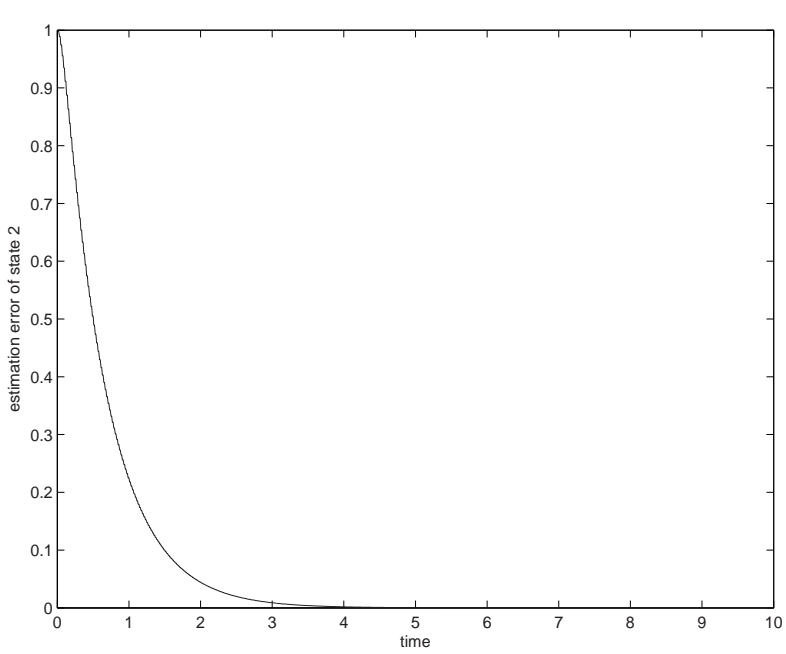

Figure 4: The estimation error of the velocity

for digital control of chaotic sysems," IEEE Transactions on Fuzzy Systems, vol. 1, pp. 394-408, 1999.

[2] H. K. Lam and F. H. F. Leung, "Sampled-data fuzzy controler for time-delay nonlinear systems: fuzzymodel-based LMI approach," IEEE Transactions on System, Man and Cybernetics B, vol. 37, no. 3 pp. 617629, 2007.

[3] H. K. Lam and W. K. Ling, "Sampled-data fuzzy controller for continuous nonlinear systems," IET Control Theory Application, vol. 2, no. 1, pp. 32-39, 2008.

[4] H. Gao and T. Chen, "Stabilization of nonlinear systems under variable sampling: a fuzzy control approach," IEEE Transactions on Fuzzy Systems, vol. 15, no. 5, pp. 972-983, 2007.

[5] W. Chang, J. B. Park, Y. H. Joo and G. Chen, "Design of sampled-data fuzzy-model-based control systems by using intelligent digital redesign," IEEE Transactions on Circuits and Systems, vol. 49, pp. 509-517, 2002.

[6] H. J. Lee, H. B. Kim, Y. H. Joo, W. Chang and J. B. Park, "A new intelligent digital redesign for TS fuzzy systems: global approach," IEEE Transactions on Fuzzy Systems, vol. 12, pp. 274-284, 2004.

[7] H. J. Lee, J. B. Park and Y. H. Joo, "Digitalizing a fuzzy observer-based output-feedback control: intelligent digital redesign approach," IEEE Transactions on Fuzzy Systems, vol. 13, pp. 701-716, 2005.

[8] H. C. Sung, D. W. Kim, J. B. Park and Y. H. Joo, "Robust digital control of fuzzy systems with parametric uncertainties: LMI-based digital redesign approach," Fuzzy Sets and Systems, vol. 161, pp. 919-933, 2010. 
[9] K. Gu, "An integral inequality in the stability problem of time-delay systems," Proc. 39th IEEE Conf. Decis. Control, pp. 2805-2810, 2000.

[10] I. R. Petersen, "A stabilization algorithm for a class of uncertain linear systems," Systems and Control Letters, vol. 8, pp. 351-357, 1987.

[11] L. Xie, "Output feedback $H_{\infty}$ control of systems with parameter uncertainties," Int. J. Contr, vol. 63, no. 4, pp. 741-750, 1996.

[12] H. Luan, O. C. Onar, and A. Khaligh, "Dynamic modeling and optimum load control of a PM linear generator for ocean wave energy harvesting application," Applied Power Electronics Conference and Exposition, 2009, pp. 739-743, 2009.

[13] P. Meisen and T. Hammons, "Harnessing the untapped energy potential of the oceans: tidal, wave, currents and otec," IEEE Power engineering socity energy development and power generation committee, pp. 1853-1854, 2005.

\section{Geun Bum Koo}

Ph.D course student of Yonsei University

Research Area: Fuzzy control, Interconnected system, Sampled-data control, etc.

E-mail : milbam@yonsei.ac.kr

\section{Jin Bae Park}

Professor of Yonsei University

Research Area: Control theory, Filtering theory, Robotics, Automation, Hadamard transform, MEMS, etc.

E-mail : jbpark@yonsei.ac.kr

\section{Young Hoon Joo}

Professor of Kunsan National University

Research Area: Intelligent robot system control, Intelligent control and modeling, Nonlinear system control, Intelligent emotion recognition, WEB-based virtual laboratory, etc.

E-mail : yhjoo@kunsan.ac.kr 